\title{
Mindfulness training for chronic fatigue syndrome: a pilot study
}

\begin{abstract}
BACKGROUND
Chronic fatigue syndrome (CFS) is a condition characterized by persistent and unexplained fatigue that may result in severe impairment of daily functioning. Currently, there is no curative treatment for CFS, and many patients experience the existing interventions as ineffective. Thus, there is a need for new approaches that target psychological maintenance factors and coping. Mindfulness is an approach to increasing awareness and acceptance of ongoing mental processes. Mindfulness-based interventions have been shown to reduce stress and enhance quality of life in patients with chronic diseases, to increase the tolerance of unpleasant feelings and bodily dysfunction, as well as to facilitate use of appropriate coping skills. This pilot study examined the effect and acceptability of a mindfulness-based intervention for patients with CFS.
\end{abstract}

PARTICIPANTS AND PROCEDURE

Ten patients with CFS (eight women, two men) participated in the study. The mindfulness training had a duration of eight weeks with 2-hour weekly meetings. The effect of the intervention was evaluated using a single case series design with a 3-month follow-up.

\section{RESULTS}

All patients completed the intervention. Medium to large effect sizes were found for anxiety, fatigue, rumination, depression, and mindfulness. The participants' feedback indicated increased quality of life and more adaptive coping.

\section{CONCLUSIONS}

It is concluded that mindfulness-based interventions have a potential to improve the condition of patients with CFS.

\section{KEY WORDS}

mindfulness training; chronic fatigue; depression; anxiety; coping

ORGANizATION - 1: Oslo University Hospital, Oslo, Norway · 2: Department of Psychology, University of Tromsø, Tromsø, Norway

authors' Contributions - A: Study design - B: Data collection - C: Statistical analysis - D: Data interpretation -

E: Manuscript preparation · F: Literature search · G: Funds collection

Corresponding Author - Jens C. Thimm, Ph.D., Department of Psychology, University of Tromsø, 9037 Troms $\varnothing$,

Norway, e-mail: jens.thimm@uit.no

to Cite this ARticLe - Sollie, K., Næss, E. T., Solhaug, I., \& Thimm, J. C. (2017). Mindfulness training for chronic fatigue

syndrome: a pilot study. Health Psychology Report, 5(3), 240-250. doi: https://doi.org/10.5114/hpr.2017.65469

RECEIVED 27.08.2016 · REVIEWED 28.09.2016 · ACCEPTED 29.10.2016 · PUBLISHED 01.02.2017 


\section{BACKGROUND}

Chronic fatigue syndrome (CFS) is a condition characterized by persistent and unexplained fatigue that may result in severe impairment of daily functioning (Afaria \& Buchwald, 2003; Prins, van der Meer, \& Bleijenberg, 2006). Chronic fatigue syndrome is commonly associated with numerous somatic and cognitive problems, such as impaired concentration and short-term memory, sleep disorders, pain, immunological and neuroendocrine symptoms (Carruthers et al., 2003; Fukuda et al., 1994). Different criteria sets for the diagnosis of CFS have been developed (i.e., Brurberg, Fønhus, Larun, Flottorp, \& Malterud, 2014; Haney et al., 2015; Jason et al., 2015). Depending on the criteria used, the reported incidence of CFS varies from less than $1 \%$ to $7.60 \%$ (Brurberg et al., 2014). Having had CFS for a period longer than 15 months is associated with a worse prognosis (Johnson, 2013), whereas patients with less severe symptoms at onset seem to be more likely to recover (Johnson, 2013). Children have a better prognosis than adults (Carruthers et al., 2003; Wyller et al., 2006).

Due to the lack of distinct biological markers, CFS is often categorized as a somatization disorder (e.g., Lakhan \& Schofield, 2013) or as medically unexplained symptoms (e.g., Deary, Chalder, \& Sharpe, 2007). The diagnosis of CFS can be difficult to determine (Brurberg et al., 2014; Haney et al., 2015) and is based on exclusion (e.g., Fukuda et al., 1994). It is assumed that CFS can have different or multiple causes (Afaria \& Buchwald, 2003). Both psychological, biological, and immunological explanations are proposed (Johnson, 2013; Prins et al., 2006). A distinction is usually further made between predisposing, triggering, and maintaining factors (Prins et al., 2006). Predisposing factors can be personality, lifestyle, or genetic risk factors. Triggers can be acute physical and psychological stress, infection, or serious life events. Maintenance factors can be psychological mechanisms, such as dysfunctional thought and behavior patterns that contribute to maintain fatigue (Prins et al., 2006).

No curative treatment for CFS is currently available (Johnson, 2013). A recent systematic review concluded that patients benefit from pharmacological treatment with rintatolimod, psychological interventions, and graded exercise (Smith et al., 2015). However, the effects of pharmacological treatments are small, and the findings about psychological interventions are inconsistent (Smith et al., 2015). In clinical practice, only $18 \%$ of patients show complete recovery (Flo \& Chalder, 2014). A recent user survey of 828 CFS patients in Norway (Bjørkum, Wang, \& Waterloo, 2009) showed that $97 \%$ of patients considered pacing, rest, complete shielding, and quietness as useful. Cognitive-behavioral therapy (CBT) was con- sidered effective by $57 \%$. Notably, $79 \%$ reported that graded exercise led to a deterioration of symptoms (Bjørkum et al., 2009). Thus, there is a need for new approaches for these patients that target factors that are shown to maintain or worsen CFS symptoms. For example, findings indicate that the ability to accept the conditions related to CFS and to acquire adaptive coping skills is associated with increased quality of life (Brooks, Rimes, \& Chalder, 2011; Van Damme, Crombez, Van Houdenhove, Mariman, \& Michielsen, 2006). Moreover, illness acceptance has been shown to have an important role in the psychological treatment of CFS (Poppe, Petrovic, Vogelaers, \& Crombez, 2013). Conversely, self-critical perfectionism has been shown to predict increased fatigue and pain (Kempke et al., 2013). Hence, CFS patients may benefit from interventions that enhance awareness and acceptance.

Mindfulness originates from eastern philosophy and practice and has during the last decades been translated into secular, evidence-based clinical practice (Williams, Teasdale, Segal, \& Kabat-Zinn, 2007). Being mindful can be described as being consciously aware of present moment experience. A non-judgmental and accepting attitude towards experiences is considered a basic element of mindfulness-based interventions and an important mechanism for change (Baer, 2003; Roemer \& Orsillo, 2003; Williams et al., 2007). Mindfulness is an approach for increased awareness of and deliberate response to habitual mental processes that contribute to emotional stress and maladaptive behavior (Bishop et al., 2004).

Mindfulness-based interventions, such as mindfulness-based stress reduction (MBSR; Kabat-Zinn, 1990) and mindfulness-based cognitive therapy (MBCT; Segal, Williams, \& Teasdale, 2013), have shown positive results among patients with stress-related symptoms, pain disorders, mental disorders, and a variety of chronic diseases (Gotink et al., 2015; Goyal et al., 2014). Physical health benefits of MBSR have been reported for sleep quality, fatigue, general quality of life, and somatic complaints (Baer, 2003; Garmon, Philbrick, Padrick, \& Goodman, 2014; Grossman, Thifenthaler-Gilmer, Raysz, \& Kesper, 2007; Merkes, 2010). MBCT has been shown to be effective for anxiety disorders and recurrence of depressive disorders (Hofmann, Sawyer, Witt, \& Oh, 2010; Khoury et al., 2013).

Preliminary findings suggest that mindfulness-based interventions can be beneficial for patients with CFS (e.g., Fjorback et al., 2013; Rimes \& Wingrove, 2013; Surawy, Roberts, \& Silver, 2005). In a series of three exploratory studies, Surawy et al. (2005) evaluated a mindfulness-based intervention based on MBSR and MBCT and found significant improvements in fatigue, anxiety, depression, quality of life, and physical functioning that were maintained at 3-month follow-up. Fjorback et al. (2013) tested in
Mindfulness training for CFS 
Katinka Sollie, Eva Therese Næss, Ida Solhaug, Jens C. Thimm a randomized trial the efficacy of mindfulness therapy in a sample of patients with somatization disorder and functional somatic syndromes, $74 \%$ of whom met the criteria for CFS. The control group received treatment as usual enhanced by an individualized CBT treatment plan. The results showed significant reductions in the SF-36 Physical Component Summary, health anxiety, physical symptoms, anxiety, and depression in the mindfulness therapy group with effect sizes (Cohen's $d$ ) ranging from .27 (physical symptoms at posttest) to .80 (health anxiety at 15-month follow-up). However, at 15-month follow up, the differences between the mindfulness group and the control group were nonsignificant. Rimes and Wingrove (2013) conducted a randomized pilot study in which they measured the effect of an 8-week MBCT intervention for patients with CFS who still experienced abnormal high fatigue levels after treatment with CBT. The intervention was associated with a reduction of fatigue, maintained at 2- and 6-month follow-up, as well as effects on disability, depressive mood, mindfulness, and self-compassion. While these results are promising, clearly more research on mindfulness-based interventions for patients with CFS is warranted.

The present study's aim was to add to the limited field of knowledge with respect to the effects of mindfulness-based interventions for patients with CFS. To accomplish this, we developed and tested an 8-week mindfulness-based intervention for patients with CFS, adapted from the MBCT program (Næss \& Solhaug, 2013). In this pilot study, we aimed at investigating the intervention's impact on both CFS symptomatology and mental health and quality of life. In addition, we aimed to evaluate the acceptability of the intervention. Thus, we posed the following hypotheses:

a) The intervention decreases CFS symptomatology.

b) The intervention reduces anxiety, depression, and rumination, and enhances quality of life and trait mindfulness.

c) The mindfulness intervention is acceptable to CFS patients.

\section{PARTICIPANTS AND PROCEDURE}

\section{DESIGN}

A single case series with an A-B design and a 3-month follow-up was used (Barlow, Nock, \& Hersen, 2009).

\section{PARTICIPANTS}

Eight women and two men participated. The mean age was 43.50 years ( $S D=9.90$, range: $31-58$ years). Nine participants were recruited by an advertise- ment in a local newspaper. The last participant was recruited by another participant in a meeting of a local support group for CFS patients. Inclusion criteria were a diagnosis of CFS based on the Canada criteria (Carruthers et al., 2003), age above 18 years, and sufficient Norwegian language skills. Previous experience with mindfulness-based interventions was not an exclusion criterion.

The participants had had symptoms of CFS for an average of 9.80 years, ranging from one to 19 years. They had received the diagnosis on average 5.70 years ago with a range from six months to 16 years. Eight participants reported that they had been diagnosed based on the Canada criteria (Carruthers et al., 2003). Two participants did not know which diagnostic criteria were used.

Of the ten participants, one was actively using mindfulness, two had previously participated in mindfulness training (one and five years ago, respectively) and one had practiced yoga. The remaining participants had no previous experience with mindfulness training. One participant was working fulltime, and another was under a rehabilitation process. The rest of the participants were on sick leave or received a disability pension. One patient was pregnant during the study period. Four participants received no other treatment parallel to the intervention. The remaining participants tried other treatments simultaneously: medication with LDN (low-dose naltrexone), psychomotoric therapy, physiotherapy, acupuncture, or diet changes.

\section{INTERVENTION}

The intervention was based on MBCT (Segal et al., 2013) and adapted for individuals with CFS. The intervention had a duration of eight weeks with 2-hour weekly meetings.

The intervention comprised different exercises aiming at enhancing the ability to observe bodily sensations, thoughts, and feelings in an accepting and non-judgmental way. To fit the current population, the exercises and movements were shortened and less energy demanding. One goal was that the participants learned to identify their window of tolerance and gained more insight into their mental, emotional, and physical boundaries and possibilities, as well as to regulate activity concerning these experiences. As a part of the mindfulness intervention, the participants received a workbook containing instructions, explanations, and homework, as well as a CD with mindfulness-based meditations and yoga exercises. The participants received one module from the workbook at every meeting. A more comprehensive description of the intervention can be found in the instructor manual (Næss \& Solhaug, 2013). 
The intervention was developed and conducted by Ida Solhaug and Eva Therese Næss, who had received training in MBSR and/or MBCT from acknowledged international institutions and had been practicing mindfulness for several years. IS has been an instructor for mindfulness-based groups for ten years. ETN is a clinical psychologist and experienced group therapist.

\section{MEASURES}

The participants' fatigue was assessed with the Chalder Fatigue Scale (CFS; Chalder et al., 1993). The CFS has 11 items that are answered on a four-point Likert scale from 0 (less than usual) to 3 (much more than usual). The scale has adequate psychometric properties (Cella \& Chalder, 2010) and has previously been used in research in Norway (e.g., Flo \& Chalder, 2014). Flo and Chalder (2014) reported a Cronbach's $\alpha$ of .92 of the Norwegian CFS.

We wanted to assess the participants' symptom burden in accordance with the Canada criteria for CFS (Carruthers et al., 2003). As there are currently no instruments in Norwegian available, a self-report inventory was constructed. The scale had 30 items that are answered on a five-point Likert scale from 0 (not at all) to 4 (very much) and cover the following domains: fatigue, feelings of illness, exhaustion after exertion, sleep problems, pain, and neurological, cognitive, autonomous, neuroendocrine, and immunological manifestations. An average score of all items was calculated.

The participants' level of anxiety and depression was assessed with the Hospital Anxiety and Depression Scale (HADS) (Zigmond \& Snaith, 1983). The HADS is an inventory with 14 items that are answered on a fourpoint Likert scale from 0 to 3 . The scale was developed to avoid the effects of somatic diseases such as exhaustion, insomnia, and dizziness (Bjelland, Dahl, Haug, \& Necklemann, 2001). The scale is therefore particularly suited for the assessment of anxiety and depression in patients with somatic health problems. Øyane, Pallesen, Moen, Åkerstedt, and Bjorvatn (2013) reported a Cronbach's $\alpha$ of .82 for both the anxiety and depression scales of the Norwegian version of the HADS.

The participants' tendency to ruminate was measured with the Ruminative Response Scale (RRS; Nolen-Hoeksema \& Morrow, 1991). This self-report scale comprises 22 items that are scored on a fourpoint Likert scale from 1 (almost never) to 4 (almost always). Several studies have supported the reliability and validity of the RRS (Luminet, 2004). The RRS has previously been used in research in Norway (e.g., Halvorsen et al., 2015). In the Halvorsen et al. (2015) study, the RRS had a Cronbach's $\alpha$ of .95 .

The participants' dispositional mindfulness was assessed with the Five Facet Mindfulness Question- naire (FFMQ; Baer, Smith, Hopkins, Krietemeyer, $\&$ Toney, 2005). The FFMQ has 39 items that are answered on a five-point Likert scale from 1 (never or very rarely true) to 5 (very often or always true). The five facets are observing, describing, acting with awareness, nonjudging of inner experience, and nonreactivity to inner experience (Baer et al., 2006). The FFMQ has been validated in Norway (Dundas, Vøllestad, Binder, \& Sivertsen, 2013). In the present study the FFMQ total score was used. For the total score of the Norwegian FFMQ, Dundas et al. (2013) found a Cronbach's $\alpha$ of .86 .

Diener, Emmons, Larsen, and Griffin's (1985) Satisfaction With Life Scale (SWLS) was used to assess the participants' quality of life. The scale consists of seven items that are rated on a 7-point Likert scale from 1 (strongly disagree) to 7 (strongly agree). The scale has been validated in Norway (Clench-Aas, Nes, Dalgard, \& Aarø, 2011). Clench-Aas et al. (2011) reported a Cronbach's $\alpha$ of .91 for the Norwegian version of the SWLS.

The participants' evaluation of the intervention was anonymously assessed with a form containing questions about satisfaction, gains, and which components and exercises they experienced as useful. At 3-month follow-up, the participants completed a form concerning their use of mindfulness in the last four weeks.

\section{PROCEDURE}

Before the start of the intervention, the group leaders met the participants individually to give information about the project as well as to provide an introduction to the intervention. An additional purpose of this meeting was to prevent absence from meetings/ drop-out and to increase motivation.

The baseline measures (CFS, HADS, RRS, symptom burden) were sent to the participants three times with approximately one-week intervals in the month before the intervention. After the first group meeting, the pretest was distributed. After the last meeting, the posttest was administered. A follow-up was conducted three months after the last group session. At pretest, posttest, and follow-up, the SWLS and FFMQ were included in addition to the baseline measures. The study has been approved by the Regional Committee for Medical and Health Research Ethics.

\section{STATISTICAL ANALYSES}

Due to the small sample size, it is inexpedient to use confirmatory statistics. The size of the treatment effects are reported as Cohen's $d$ for within-group change. T-statistics for dependent samples were used to calculate $d(t / \sqrt{N})$. The effect sizes were interpreted as small
Mindfulness training for CFS 
$(d=.20)$, medium $(d=.50)$, and large $(d=.80)$ according to Cohen (1988). With regard to the individual participants, a $50 \%$ change in score was considered clinically significant (cf. Hiller, Schindler, \& Lambert, 2012).

\section{RESULTS}

\section{GROUP-BASED MEANS}

Katinka Sollie, Eva Therese Næss, Ida Solhaug, Jens C. Thimm

All participants completed the intervention and answered the pretest, posttest, and follow-up measures. However, the number of baseline data points varied between the participants from one to three. Visual inspection suggested that the baseline measures were stable overall despite some variations. Table 1 displays means, standard deviations, and effect sizes of the outcome measures at pretest, posttest, and follow-up. The participants' scores at the different time points are shown in Figure 1.

As shown in Table 1, effect sizes between pretest and posttest were medium to large for anxiety (.68) and fatigue (.56), and small to medium for depression (.32) and rumination (.26). At follow-up, compared to pretest the effect sizes were medium to large for mindfulness (.77) and anxiety, and small to medium for depression (.33), rumination (.32) and fatigue (.26).

\section{CLINICAL SIGNIFICANCE}

One participant reported significantly reduced fatigue from pretest to posttest and another participant from pretest to follow-up. Four participants showed a significant reduction in anxiety from pretest to posttest and three participants at follow-up. Three participants experienced a significant reduction in depressive symptoms from pretest to posttest that was maintained at follow-up. One participant showed a significant increase of depressive symptoms at posttest and follow-up compared to the pretest. However, the participants' scores were in the normal range at all three time points. None of the participants reported a reduction in rumination at posttest or follow-up larger than $50 \%$. One participant had a significant reduction of symptom burden, but two participants reported a deterioration of CFS symptoms at posttest, one of them also at follow-up. The latter participant though had a very low score at pretest (.03) and a significantly elevated, but still low score at posttest (.13) and follow-up (.67). None of the participants showed a significant change in mindfulness. One participant experienced a significant decrease in quality of life at posttest and follow-up.

\section{ACCEPTABILITY}

The average satisfaction was $80 \%$ with a range from $72 \%$ to $88 \%$. Three participants reported that they felt improved in their condition since the start of the intervention. One participant experienced deterioration of symptoms but related this to an ongoing food intolerance test, and not the intervention. The remaining participants reported neither an improvement nor a worsening of their condition after the intervention.

Some participants reported that the best aspect of the course had been that they learned energy conservation, which increased their quality of life. One participant reported that the course had not reduced her pain, but helped her to cope better. Several participants reported that mindfulness had helped them to appreciate what is good in life and given them the ability to feel pleasure. Some participants stated that mindfulness provides a different kind of rest that has a positive physical effect.

Table 1

Means, standard deviations, and effect sizes (Cohen's d) of the study measures at pretest, posttest, and 3-month follow-up

\begin{tabular}{|c|c|c|c|c|c|}
\hline & $\begin{array}{l}\text { Pretest } \\
M(S D)\end{array}$ & $\begin{array}{c}\text { Posttest } \\
M(S D)\end{array}$ & $\begin{array}{l}\text { Follow-up } \\
\qquad M(S D)\end{array}$ & $\begin{array}{c}\text { ES Pretest- } \\
\text { posttest } \\
d\end{array}$ & $\begin{array}{c}\text { ES Pretest- } \\
\text { follow-up } \\
d\end{array}$ \\
\hline Fatigue scale & $21.70(6.52)$ & $19.60(7.72)$ & $20.60(8.95)$ & .56 & .26 \\
\hline HADS anxiety & $6.10(5.13)$ & $3.30(2.71)$ & $4.20(4.13)$ & .68 & .48 \\
\hline HADS depression & $4.30(3.68)$ & $3.10(2.60)$ & $2.90(2.92)$ & .32 & .33 \\
\hline RRS & $33.50(10.23)$ & $31.00(5.00)$ & $30.80(5.25)$ & .26 & .32 \\
\hline CFS symptom burden & $1.79(1.12)$ & $1.73(0.84)$ & $1.75(0.92)$ & .07 & .04 \\
\hline FFMQ & $113.20(10.88)$ & $114.60(7.85)$ & $118.80(10.41)$ & .11 & .77 \\
\hline SWLS & $24.70(10.11)$ & $24.10(9.15)$ & $25.60(8.86)$ & -.09 & .09 \\
\hline
\end{tabular}

Note. ES - effect size; $d$ - Cohen's d; HADS - Hospital Anxiety and Depression Scale; RRS - Ruminative Response Scale; FFMQ Five Facet Mindfulness Questionnaire; SWLS - Satisfaction With Life Scale. 
Participant 1
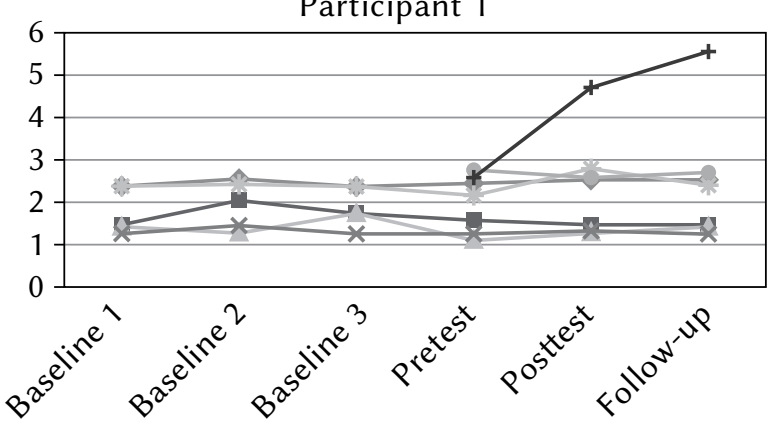

Participant 3

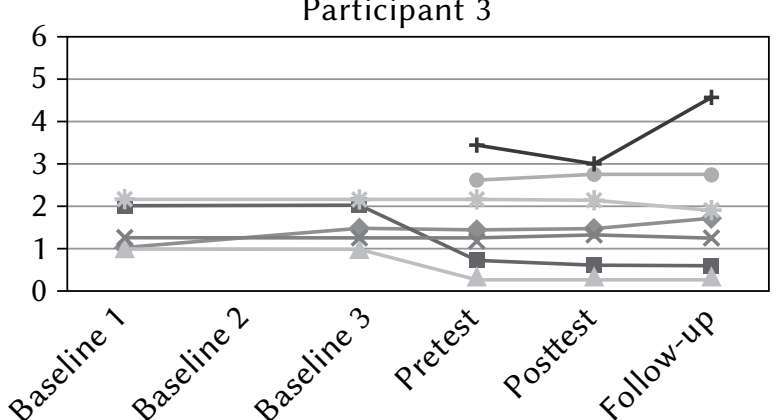

Participant 5
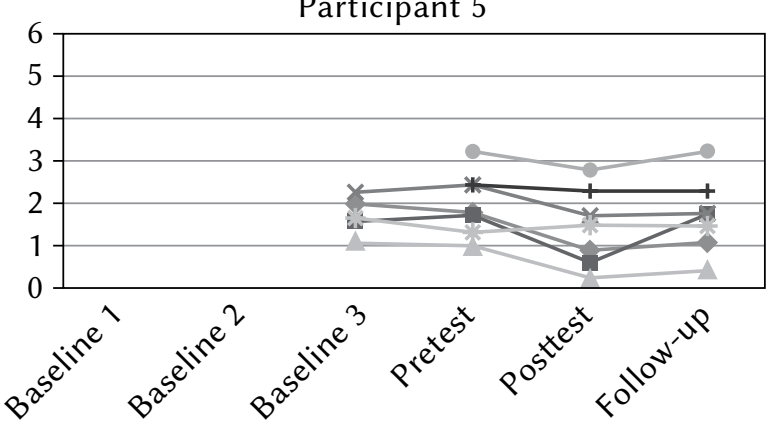

Participant 7
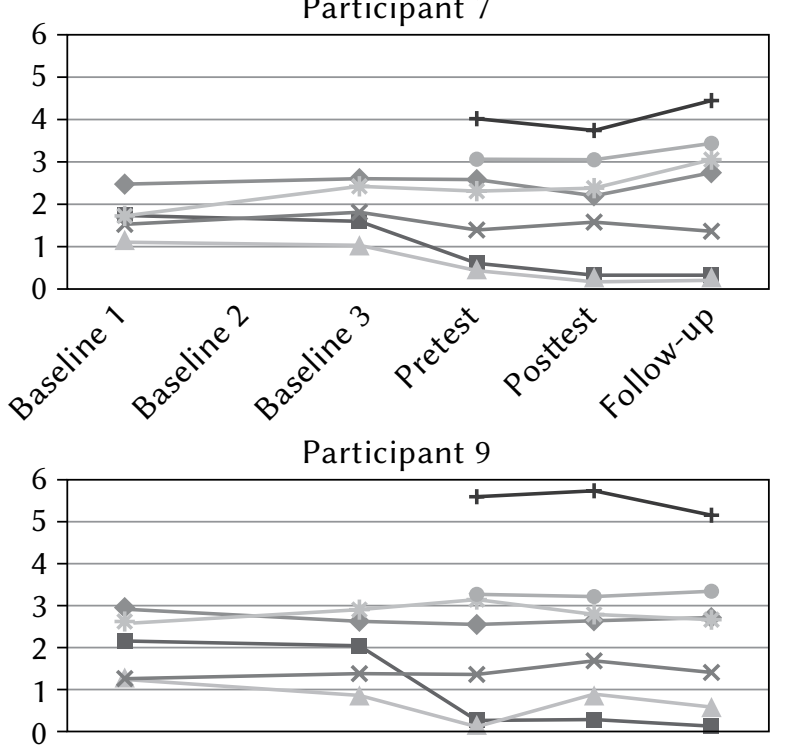

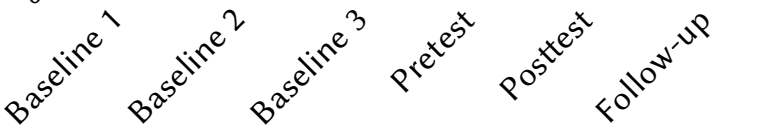

Participant 2

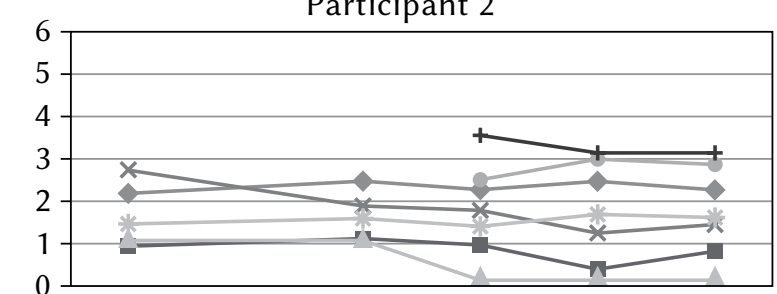

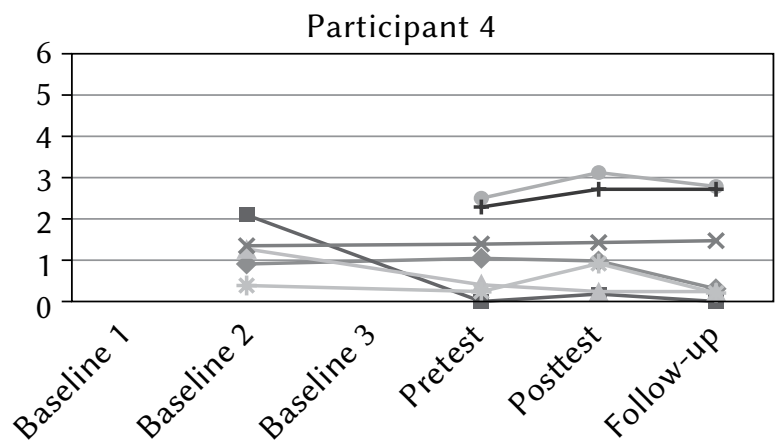

Mindfulness

training for CFS

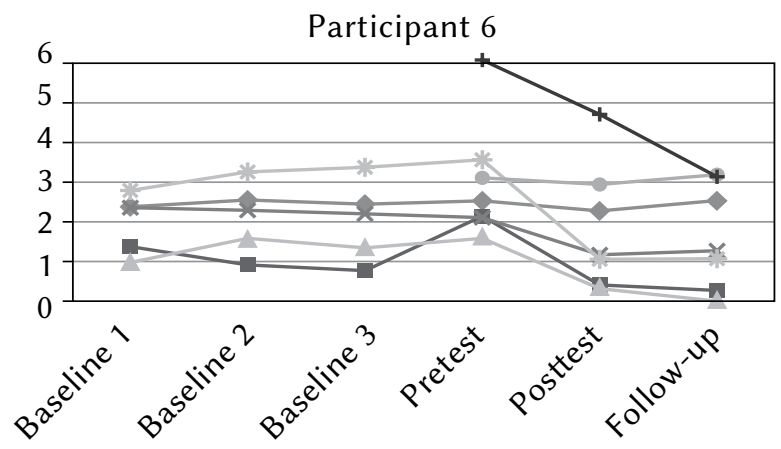

Participant 8

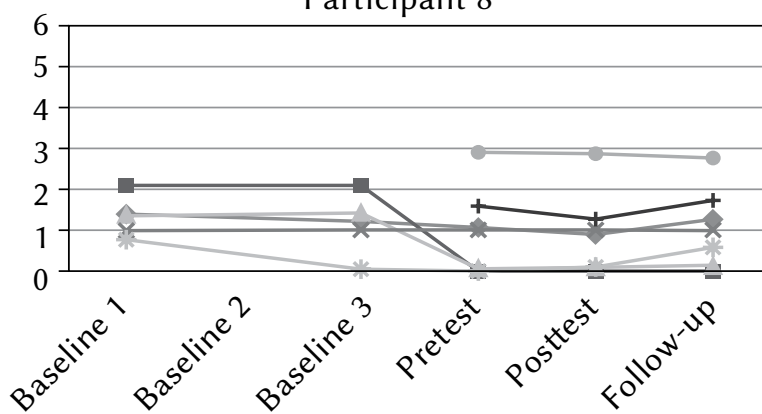

Participant 10

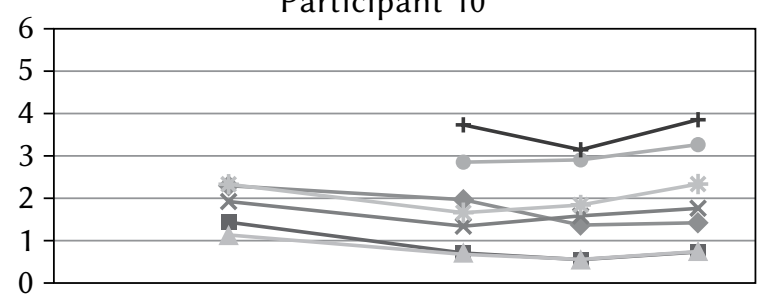

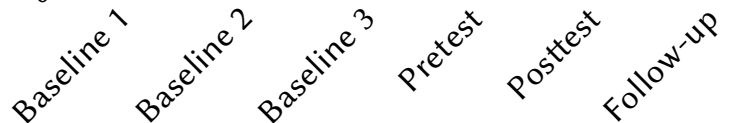

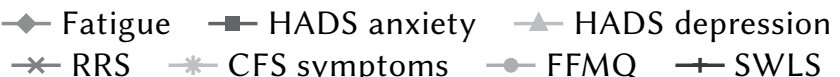

Figure 1. Mean item scores on the Fatigue scale, HADS anxiety, HADS depression, Ruminative Response Scale (RRS), CFS symptom burden, Five Facet Mindfulness Questionnaire (FFMQ), and Satisfaction With Life Scale (SWLS) from baseline through pre-treatment, post-treatment, and follow-up. 
Katinka Sollie, Eva Therese Næss, Ida Solhaug, Jens C. Thimm
The participants were asked to rank the usefulness of the different techniques used in the course. "Body scanning with CD instructions" and "attention to breath in daily life" received the highest average scores (91\%). After that followed "group discussions" (82\%), "yoga exercises with instruction" (74\%) and "awareness exercise with CD instruction while sitting” (71\%). Techniques without using the CD instruction received slightly lower scores. "Self-kindness meditation" (66\%), "body scan without CD instruction" (62\%), "attention exercise without CD instruction while sitting" $(60 \%)$ and "workbook and own notes" (59\%) were perceived as beneficial by some, but not all participants. All tools except "yoga exercises without CD instruction" (49\%) had an average usefulness score of over $50 \%$. The greatest barriers for the participants to use the tools they had learned in the course were time, space, energy, and implementing new routines.

At follow-up, eight participants reported that they had practiced mindfulness in the last four weeks, five of them daily, one participant 4-6 times per week, another participant 1-3 times per week, and the last participant once.

\section{DISCUSSION}

A mindfulness-based intervention adapted for patients with CFS showed a positive effect on anxiety, fatigue, depression, and rumination at posttest, with small to moderate effect sizes. At follow-up, the effect increased for dispositional mindfulness, was maintained for depression and rumination, and slightly decreased for fatigue and anxiety. The results showed no impact on a composite measure of CFS symptomatology and general quality of life. Overall, these findings are in line with previous research (e.g., Fjorback et al., 2013; Rimes \& Wingrove, 2013; Surawy et al., 2005).

The reduction in perceived fatigue among participants aligns with previous mindfulness-based intervention adapted for CFS (Rimes \& Wingrove, 2013; Surawy et al., 2005). Feedback from participants supports these findings, indicating that participants experience a "different kind of rest" when they practice mindfulness, providing "more energy" than if they were sleeping or just lying down during the day. Thus, the participants seem to have benefitted from the relaxing effect of mindfulness (Hölzel et al., 2011). Moreover, increased body awareness (cf. Hölzel et al., 2011) and more accurate perception of physical symptoms (Mirams, Poliakoff, Brown, \& Lloyd, 2013) through the mindfulness training may have facilitated energy conservation.

The participants' levels of anxiety and depressive symptoms were reduced in the course of the intervention. As CFS is often comorbid with depres- sion and/or anxiety (Afari \& Buchwald, 2003; Cella, White, Sharpe, \& Chalder, 2013), the reduction of such symptoms is an important concern. This finding aligns with previous research on mindfulness-based interventions reporting reduced levels of anxiety and depression (e.g., Bohlmeijer, Prenger, Taal, \& Cuijpers, 2010; Bondolfi et al., 2010; Fjorback et al., 2013; Segal et al., 2013; Surawy et al., 2005; Rimes \& Wingrove, 2013). It has been hypothesized that mindfulness-based interventions aid participants in changing a brooding thought pattern to a more attentive and accepting presence (Rimes \& Wingrove, 2013). In support of such a hypothesis, our participants showed a slight reduction in rumination from pretest to posttest which was maintained at follow-up. Thus, mindfulness might enhance consciousness of automatic thought patterns and facilitate greater response flexibility, contributing to decreased levels of rumination and depression, as previous research indicates (i.e., Britton, Shahar, Szepsenwol, \& Jacobs, 2012).

Contrary to the findings of Surawy et al. (2005), the intervention had no effect on general quality of life as measured by the SWLS. Similarly, the intervention showed no effect on dispositional mindfulness at posttest. One possible explanation is that most participants have had their diagnosis over many years. Maladaptive coping strategies may have persisted for some time, and the integration of new thought and behavioral patterns may require more time. Both participants and group leaders expressed that they would have preferred a longer duration of the course, and it is conceivable that the mindfulness attitudinal and attentional components had not been well enough integrated. In accordance with this, van Ravesteijn et al. (2014) suggested that an MBCT course can start a process of awareness and acceptance that needs consolidation. The effect on dispositional mindfulness, however, increased considerably at follow-up which may be a result of most participants continuing mindfulness training after the course. Future research on mindfulness-based interventions with longer durability and long-term follow-up are encouraged.

The user satisfaction with this intervention was high (80\%) and comparable to previous studies (Rimes \& Wingrove, 2013). In line with this finding, feedback from the participants indicated that they felt grateful for taking part in the program, highlighting that they felt that they had learned new techniques to master everyday life. Some participants stated that they "are not improved, but feel better", which indicates improved quality of life and changed attitudes towards their condition even if the symptoms of fatigue still might prevail. The seeming discrepancy between these reports and the results of the self-report inventories suggests that the quantitative methods may not have fully captured the subjective experience of 
each participant after course participation. Future studies should include more rigorous qualitative assessments of the participants' subjective experience of the intervention or other measures of quality of life or psychosocial functioning.

In summary, the results of this pilot study align with the existing literature on mindfulness-based therapies for somatization disorders (e.g., Lakhan \& Schofield, 2013) and chronic medical conditions (e.g., Bohlmeijer et al., 2010) in general and CFS in particular (e.g., Rimes \& Wingrove, 2013; Surawy et al., 2005), indicating positive effects on fatigue, depression, and anxiety with small to medium effect sizes. The intervention is highly acceptable for the patients, and no harmful side effects were observed.

The current investigation has several weaknesses, first and foremost the lack of a control group. By including a control group one will be able to be more certain that observed changes can actually be attributed to the intervention and are not due to nonspecific factors such as group cohesion or the therapists' enthusiasm (cf. Davidson \& Kaszniak, 2015). In addition, the sample size of this study was small. A possible replication could benefit from a larger sample, spread over several groups, to increase generalizability and statistical power. Finally, the sample was heterogeneous in terms of familiarity with mindfulness training and duration of symptom presence. Four patients had previously participated in a mindfulness intervention or were using mindfulness exercises actively. It is conceivable that participants who are familiar with mindfulness practice benefit less from the current intervention than those who have no previous experience with mindfulness training. Thus, the inclusion of participants with previous mindfulness experience in the current study may have led to an underestimation of the intervention's effect. On the other hand, the unselected sample is presumably representative of CFS patients encountered in routine clinical practice.

Based on the results of self-report measures of symptoms, positive feedback from the participants and no drop-outs, the findings of this pilot study indicate that mindfulness-based interventions are beneficial for patients diagnosed with CFS. However, these results are not conclusive, and further and more rigorous research designs are warranted in this field. The findings encourage future and larger-scale research on mindfulness-based interventions for this patient group.

\section{REFERENCES}

Afari, N., \& Buchwald, D. (2003). Chronic Fatigue Syndrome: A Review. American Journal of Psychiatry, 160, 221-236. doi: 10.1176/appi.ajp.160.2.221

Baer, R. A. (2003). Mindfulness training as a clinical intervention: A conceptual and empirical review.
Clinical Psychology: Science and Practice, 10, 125145. doi: 10.1093/clipsy/bpg015

Baer, R. A., Smith, G. T., Hopkins, J., Krietemeyer, J., \& Toney, L. (2006). Using self-report assessment methods to explore facets of mindfulness. Assessment, 13, 27-45. doi: 10.1177/1073191105283504

Barlow, D. H., Nock, M. K., \& Hersen, M. (2009). Single case experimental designs ( $3^{\text {rd }}$ ed.). Boston: Pearson Education.

Bishop, S. R., Lau, M., Shapiro, S., Carlson, L., Anderson, N. D., Carmody, J., Segal, Z. N., Abbey, S., Speca, M., Velting, D., \& Devins, G. (2004). Mindfulness: a proposed operational definition. Clinical Psychology: Science and Practice, 11, 230-241. doi: 10.1093/clipsyftph077

Bjelland, I., Dahl, A. A., Haug, T. T., \& Neckelmann, D. (2002). The validity of the Hospital Anxiety and Depression Scale - An updated literature review. Journal of Psychosomatic Research, 52, 69-77. doi: 10.1016/s0022-3999(01)00296-3

Bjørkum, T., Wang, C. E., \& Waterloo, K. (2009). Pasienterfaringer med ulike tiltak ved kronisk utmattelsessyndrom. Tidsskrift for Den Norske Legeforeningen, 12, 1214-1216. doi: 10.4045/tidsskr.09.35791

Bohlmeijer, E., Prenger, R., Taal, E., \& Cuijpers, P. (2010). The effects of mindfulness-based stress reduction therapy on mental health of adults with a chronic medical disease: A meta-analysis. Journal of Psychosomatic Research, 68, 539-544. doi: 10.1016/j.jpsychores.2009.10.005

Bondolfi, G., Jermann, F., Linden, M. V. D., Gex-Fabry, M., Bizzini, L., Rouget, B. W., Myers-Arrazola, L., Gonzalez, C., Segal, Z., Aubry, J. M., \& Bertschy, G. (2010). Depression relapse prophylaxis with mindfulness-based cognitive therapy: Replication and extention in the Swiss health care system. Journal of Affective Disorders, 122, 224231. doi: 10.1016/j.jad.2009.07.007

Britton, W. B., Shahar, B., Szepsenwol, O., \& Jacobs, W. J. (2012). Mindfulness-based cognitive therapy improves emotional reactivity to social stress: Results from a randomized controlled trial. Behavior Therapy, 43, 365-380. doi: 10.1016/j.beth.2011.08.006

Brooks, S. K., Rimes, K. A., \& Chalder, T. (2011). The role of acceptance in chronic fatigue syndrome. Journal of Psychosomatic Research, 71, 411-415. doi: 10.1016/j.jpsychores.2011.08.001

Brurberg, K. G., Fønhus, M. S., Larun, L., Flottorp, S., \& Malterud, K. (2014). Case definitions for chronic fatigue syndrome/myalgic encephalomyelitis (CFS/ME): A systematic review. BMJ Open, 4, e003973. doi: 10.1136/bmjopen-2013-003973

Carruthers, B. M., Jain, A. K., Meirleir, K. L., Peterson, D. L., Nancy G, K., Lerner, A. M., Bested, A. C., Flor-Henry, P., Joshi, P., Powles, A. C. P., Sherkey, J. A., \& Sande, M. I. V. D. (2003). Myalgic encephalomyelitis/chronic fatigue syndrome:
Mindfulness training for CFS 
Katinka Sollie, Eva Therese Næss, Ida Solhaug, Jens C. Thimm
Clinical working case definition, diagnostic and treatment protocols. Journal of Chronic Fatigue Syndrome, 11, 7-115. doi: 10.1300/J092v11n01_02

Cella, M., \& Chalder, T. (2010). Measuring fatigue in clinical and community settings. Journal of Psychosomatic Research, 69, 17-22. doi: 10.1016/j.jpsychores.2009.10.007

Cella, M., White, P. D., Sharpe, M., \& Chalder, T. (2013). Cognitions, behaviours and co-morbid psychiatric diagnoses in patients with chronic fatigue syndrome. Psychological Medicine, 43, 375380. doi: 10.1017/S0033291712000979

Chalder, T., Berelowitz, G., Pawlikowska, T., Watts, L., Wessely, S., Wright, D., \& Wallace, E. P. (1993). Development of a fatigue scale. Journal of Psychosomatic Research, 37, 147-153. doi: 10.1016/00223999(93)90081-p

Clench-Aas, J., Nes, R., Dalgard, O., \& Aarø, L. (2011). Dimensionality and measurement invariance in the Satisfaction with Life Scale in Norway. Quality of Life Research, 20, 1307-1317. doi: 10.1007/ s11136-011-9859-x

Cohen, J. (1988). Statistical power analysis for the behavioral sciences. Hillsdale, N. J.: Laurence Erlbaum.

Davidson, R. J., \& Kaszniak, A. W. (2015). Conceptual and methodological issues in research on mindfulness and meditation. American Psychologist, 70, 581-592. doi: 10.1037/a0039512

Deary, V., Chalder, T., \& Sharpe, M. (2007). The cognitive behavioural model of medically unexplained symptoms: A theoretical and empirical review. Clinical Psychology Review, 27, 781-797. doi: 10.1016/j.cpr.2007.07.002

Diener, E., Emmons, R. A., Larsen, R. J., \& Griffin, S. (1985). The satisfaction with life scale. Journal of Personality Assessment, 49, 71-75. doi: 10.1207/ s15327752jpa4901_13

Dundas, I., Vøllestad, J., Binder, P.-E., \& Sivertsen, B. (2013). The Five Factor Mindfulness Questionnaire in Norway. Scandinavian Journal of Psychology, 54, 250-260. doi: 10.1111/sjop.12044

Fjorback, L. O., Arendt, M., Ørnbøl, E., Walach, H., Rehfeld, E., Schröder, A., \& Fink, P. (2013). Mindfulness therapy for somatization disorder and functional somatic syndromes - randomized trial with one-year follow-up. Journal of Psychosomatic Research, 74, 31-40. doi: 10.1016/j.jpsychores.2012.09.006

Flo, E., \& Chalder, T. (2014). Prevalence and predictors of recovery from chronic fatigue syndrome in a routine clinical practice. Behaviour Research and Therapy, 63, 1-8. doi: 10.1016/j.brat.2014.08.013

Fukuda, K., Straus, S. E., Hickie, I., Sharpe, M. C., Dobbins, J. G., \& Komaroff, A. (1994). The chronic fatigue syndrome: A comprehensive approach to its definition and study. Annals of Internal Medicine, 121, 953-959. doi: 10.7326/0003-4819-121-12199412150-00009
Garmon, B., Philbrick, J., Padrick, M., \& Goodman, M. (2014). Mindfulness-based stress reduction for chronic pain: A systematic review. Journal of Pain Management, 7, 23-36.

Gotink, R. A., Chu, P., Busschbach, J. J., Benson, H., Fricchione, G. L., \& Hunink, M. G. (2015). Standardised mindfulness-based interventions in healthcare: An overview of systematic reviews and meta-analyses of RCTs. PLoS One, 10, e0124344. doi: 10.1371/journal.pone.0124344

Goyal, M., Singh, S., Sibinga, E. M., Gould, N. F., Rowland-Seymour, A., Sharma, R., Berger, Z., Sleicher, D., Maron, D. D., Shihab, H. M., Ranasinghe, P. D., Linn, S., Saha, S., Bass, E. B., \& Haythornthwaite, J. A. (2014). Meditation programs for psychological stress and well-being: A systematic review and meta-analysis. JAMA Internal Medicine, 174, 357-368. doi: 10.1001/jamainternmed.2013.13018

Grossman, P., Thifenthaler-Gilmer, U., Raysz, A., \& Kesper, U. (2007). Mindfulness: Training as an intervention for fibromyalgia: Evidence of postintervention and 3-year follow-up benefits in well being. Psychotherapy and Psychosomatics, 76, 226233. doi: $10.1159 / 000101501$

Halvorsen, M., Hagen, R., Hjemdal, O., Eriksen, M. S., Sørli, A. J., Waterloo, K., Eisemann, M., \& Wang, C. E. (2015). Metacognitions and thought control strategies in unipolar major depression: A comparison of currently depressed, previously depressed, and never-depressed individuals. Cognitive Therapy and Research, 39, 31-40. doi: 10.1007/s10608-014-9638-4

Haney, E., Smith, M. B., McDonagh, M., Pappas, M., Daeges, M., Wasson, N., \& Nelson, H. D. (2015). Diagnostic methods for myalgic encephalomyelitis/chronic fatigue syndrome: A systematic review for a national institutes of health pathways to prevention workshop. Annals of Internal Medicine, 162, 834-840. doi: 10.7326/M15-0443

Hiller, W., Schindler, A. C., \& Lambert, M. J. (2012). Defining response and remission in psychotherapy research: A comparison of the $\mathrm{RCI}$ and the method of percent improvement. Psychotherapy Research, 22, 1-11. doi: 10.1080/10503307.2011.616237

Hofmann, S. G., Sawyer, A. T., Witt, A. A., \& Oh, D. (2010). The effect of mindfulness-based therapy on anxiety and depression: A meta-analytic review. Journal of Consulting and Clinical Psychology, 78, 169-183. doi: 10.1037/a0018555

Hölzel, B. K., Lazar, S.W., Gard, T., Schuman-Olivier, Z., Vago, D. R., \& Ott, U. (2011). How does mindfulness meditation work? Proposing mechanisms of action from a conceptual and neural perspective. Perspectives on Psychological Science, 6, 537-559. doi: 10.1177/1745691611419671

Jason, L. A., So, S., Evans, M., Brown, A., Sunnquist, M., Im, Y., \& Schafer, C. (2015). An overview of op- 
erationalizing criteria for $\mathrm{ME}, \mathrm{ME} / \mathrm{CFS}$, and CFS case definitions. Journal of Prevention \& Intervention in the Community, 43, 1-4. doi: 10.1080/10852352.2014.973237

Johnson, S. K. (2013). Chronic fatigue syndrome. In M. B. Goldman, R. Troisi, \& K. M. Rexrode (eds.), Women and health ( $2^{\text {nd }}$ ed.) (pp. 1321-1330). Amsterdam: Academic Press.

Kabat-Zinn, J. (1990). Full catastrophe living: Using the wisdom of your body and mind to face stress, pain, and Illness. New York: Delta Trade.

Kempke, S., Luyten, P., Claes, S., Goossens, L., Bekaert, P., Van Wambeke, P., \& Van Houdenhove, B. (2013). Self-critical perfectionism and its relationship to fatigue and pain in the daily flow of life in patients with chronic fatigue syndrome. Psychological Medicine, 43, 995-1002. doi: 10.1017/ S0033291712001936

Khoury, B., Lecomte, T., Fortin, G., Masse, M., Therien, P., Bouchard, V., Chapleau, M. A., Paquin, K., \& Hofmann, S. G. (2013). Mindfulness-based therapy: a comprehensive meta-analysis. Clinical Psychology Review, 33, 763-771. doi: 10.1016/j. cpr.2013.05.005

Lakhan, S. E., \& Schofield, K. L. (2013). Mindfulness-based therapies in the treatment of somatization disorders: A systematic review and meta-analysis. PLoS One, 8, e71834. doi: 10.1371/ journal.pone.0071834

Luminet, O. (2004). Measurement of depressive rumination and associated constructs. In C. Papageorgiou \& A. Wells (eds.), Depressive Rumination. Nature, theory and treatment (pp. 187-216). London: Wiley.

Merkes, M. (2010). Mindfulness-based stress reduction for people with chronic diseases. Australian Journal of Primary Health, 3, 200-210. doi: 10.1071/ PY09063

Mirams, L., Poliakoff, E., Brown, R. J., \& Lloyd, D. M. (2013). Brief body-scan meditation practice improves somatosensory perceptual decision making. Consciousness and Cognition, 22, 348-359. doi: 10.1016/j.concog.2012.07.009

Nolen-Hoeksema, S., \& Morrow, J. (1991). A prospective study of depression and posttraumatic stress symptoms after a natural disaster: The 1989 loma prieta earthquake. Journal of Personality and Social Psychology, 61, 115-121. doi: 10.1037/0022-3514.61.1.115

Næss, E. T., \& Solhaug, I. (2013). Mindfulnessbasert mestring (MBM): Manual. Unpublished manuscript. Department of Psychology, University of Tromsø.

Øyane N. M. F., Pallesen S., Moen B. E., Åkerstedt T., \& Bjorvatn, B. (2013) Associations Between Night Work and Anxiety, Depression, Insomnia, Sleepiness and Fatigue in a Sample of Norwegian Nurses. PLoS One, 8, e70228. doi: 10.1371/journal. pone. 0070228
Prins, J. B., Meer, J. W., \& Bleijenberg, G. (2006). Chronic fatigue syndrome. Lancet, 367, 346-355. doi: 10.1016/S0140-6736(06)68073-2

Poppe, C., Petrovic, M., Vogelaers, D., \& Crombez, G. (2013). Cognitive behavior therapy in patients with chronic fatigue syndrome: The role of illness acceptance and neuroticism. Journal of Psychosomatic Research, 74, 367-372. doi: 10.1016/j.jpsychores.2013.02.011

Rimes, K. A., \& Wingrove, J.(2013). Mindfulness-based cognitive therapy for people with chronic fatigue syndrome still experiencing excessive fatigue after cognitive behaviour therapy: A pilot randomized study. Clinical Psychology \& Psychotherapy, 20, 107-117. doi: 10.1002/cpp.793

Roemer, L., \& Orsillo, S. M. (2003). Mindfulness: A promising intervention strategy in need of further study. Clinical Psychology: Science and Practice, 10, 172-178. doi: 10.1093/clipsy/bpg020

Segal, Z. V., Williams, M., \& Teasdale, J. D. (2013). Mindfulness-based cognitive therapy for depression. New York: Guilford Press.

Smith, M. E. B., Haney, E., McDonagh, M., Pappas, M., Daeges, M., Wasson, N., Fu, R., \& Nelson, H. D. (2015). Treatment of myalgic encephalomyelitis/ chronic fatigue syndrome: A systematic review for a national institutes of health pathways to prevention workshop treatment of myalgic encephalomyelitis/chronic fatigue syndrome. Annals of Internal Medicine, 162, 841-850. doi: 10.7326/ M15-0114

Surawy, C., Roberts, J., \& Silver, A. (2005). The effect of mindfulness training on mood and measures of fatigue, activity, and quality of life in patients with chronic fatigue syndrome on a hospital waiting list: a series of exploratory studies. Behavioural and Cognitive psychotherapy, 33, 103-109. doi: 10.1017/S135246580400181X

van Damme, S., Crombez, G., Van Houdenhove, B., Mariman, A., \& Michielsen, W. (2006). Well-being in patients with chronic fatigue syndrome: The role of acceptance. Journal of Psychosomatic Research, 61, 595-599. doi: 10.1016/j.jpsychores.2006.04.015

van Ravesteijn, H.J., Suijkerbuijk, Y. B., Langbroek, J.A., Muskens, E., Lucassen, P. L. B. J., van Weel, C., Wester, F., \& Speckens, A. E. M. (2014). Mindfulness-based cognitive therapy (MBCT) for patients with medically unexplained symptoms: Process of change. Journal of Psychosomatic Research, 77, 27-33. doi: 10.1016/j.jpsychores.2014.04.010

Williams, J. M. G., Teasdale, J. D., Segal, Z. V., \& Kabat-Zinn, J. (2007). The mindful way through depression: Freeing yourself from chronic unhappiness. New York: Guilford.

Wyller, V. B., Bjørneklett, A., Brubakk, O., Festvåg, L., Follestad, I., Malt, U., Malterud, K., Nyland, H., Rambøl, H., Stubhaug, B., \& Larun, L. (2006). Diagnostisering og behandling av kronisk utmattel-
Mindfulness training for CFS 
sessyndrom/myalgisk encefalopati (CFS/ME) $\left(2^{\text {nd }}\right.$ ed., vol. 9). Oslo: Nasjonalt kunnskapssenter for helsetjenesten, Sosial-og helsedirektoratet.

Zigmond, A. S., \& Snaith, R. P. (1983). The hospital anxiety and depression scale. Acta Psychiatrica Scandinavica, 67, 361-370. doi: 10.1111/j.16000447.1983.tb09716.x

Katinka Sollie, Eva Therese Næss, Ida Solhaug, Jens C. Thimm 